\title{
Chordoma of the lumbar spine: a case report
}

\author{
H. Tuna; V. Aydin*; M. Bozkurt and A. Attar
}

Department of Neurosurgery. Ankara University. School of Medicine. Ankara, Turkey. *Department of Neurosurgery. Adnan Menderes University. School of Medicine. Aydin, Turkey

\begin{abstract}
Summary
Chordomas are rare tumors and they may arise anywhere along the spinal column and clival bone. The vast majority of tumors are found at skull base and sacrum. Chordomas involving the lumbar spine are rare. Approximately $6 \%$ of spinal chordomas originate in the lumbar vertebrae. We report a case of this chordoma arising from the lumbar vertebra.
\end{abstract}

KEY WORDS: Chordoma. Spine. Surgery

\section{Cordoma de la columna lumbar: caso clínico}

\section{Resumen}

Los cordomas son tumores poco frecuentes que pueden presentarse en cualquier parte de la columna vertebral y en el clivus. Sólo un mínimo porcentaje de estos tumores afectan el área lumbar. En este escrito presentamos un caso de cordoma lumbar y discutimos su presentación clínica, radiológica y las opciones de tratamiento.

PALABRAS CLAVE: Cordoma. Cirugia. Columna vertebral

\section{Inroduction}

Chordomas are rare tumors, accounting for 3-4\% of all primary bone malignancies involving spine, and they are distributed throughout the midline with $50 \%$ occurring in the sacrococcygeal region, approximately $35 \%$ in the skull base, especially in the clivus, and approximately $15 \%$ in the mobile vertebral column $n^{6,10,11}$. Approximately $40 \%$ of the mobile vertebral column chordomas are observed in the lumbar region ${ }^{5}$. Chordomas arise from the remnants of primitive notochord and there is evidence of remnants of notochord and hyaline cartilage is still present in the verte-

Recibido: 18-04-04. Aceptado: 22-06-04 bral body ${ }^{14}$. In this paper we report a case of a chordoma of the lumbar spine.

\section{Case Report}

This previously healthy 58-year-oId man presented with a 1 year history of progressive back and bilateral lower-extremity pain. He reported no history of traumatic injury. His previous medical history was unremarkable. On examination, straight leg raising test was 60 degrees bilaterally.

Magnetic resonance imaging revealed L1 and L2 vertebral tumors. Tumours were located to posteror part of the both vertebral bodies and compressing the spinal cord anteriorly (Figure 1).

A L1-L2 vertebral corpectomy and total tumor excision with Th 12-L3 interbody fusion and stabilization procedure were performed (Figure 2). The patient tolerated the procedure without difficulty and experienced resolution of his back and lower extremity pain.

This patient underwent postoperative radiotherapy and at the 12-month follow-up, he was asymptomatic and free of tumor.

\section{Discussion}

Chordomas are rare, slowly growing, locally aggressive, malignant tumors arising from remnants of the notochord. Spinal chordomas are accounting for 3-4\% of all primary bone malignancies involving spine $e^{6,10}$. The incidence is approximately 0.5 per million inhabitans ${ }^{2}$. They are distributed throughout the midline with $50 \%$ occurring in the sacrococcygeal region, approximately $35 \%$ in the skull base, especially in the clivus, and approximately $15 \%$ in the mobile vertebral column ${ }^{5,11}$. When it occurs in the mobile vertebra, more than one vertebral body may be involved ${ }^{9,13}$. In our case, L1 and L2 vertebra bodies were involved. The male/female ratio is $2: 1$ for spinal chordomas.

Chordomas arise from the remnants of primitive notochord. Even though, primitive notochord disappears during the seventh week of gestation, the remnants of primitive 


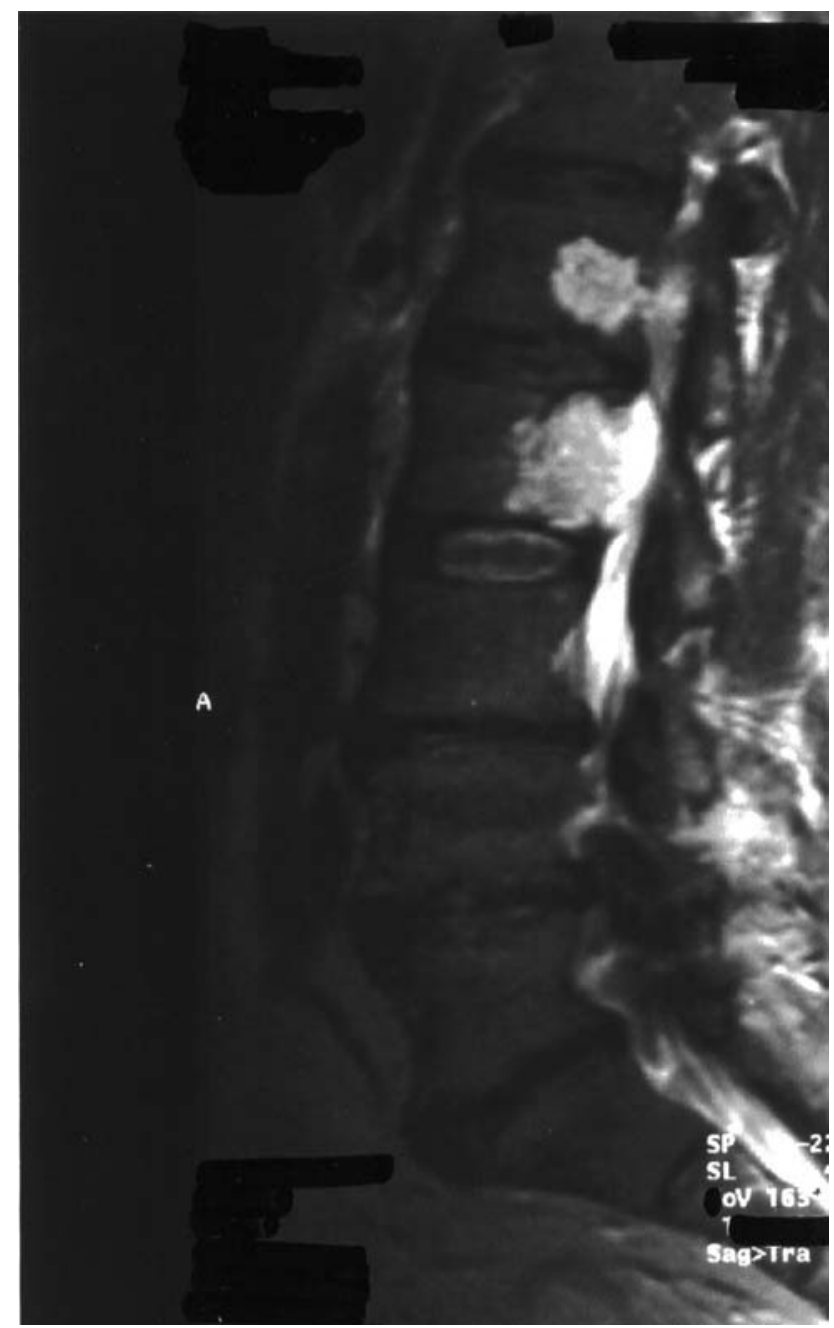

Figure 1. Preoperative sagittal T1-weighted MR with contrast enhancement image demonstrating posteriorly placed tumor at Ll and L2 vertebra bodies and extensive obstruction of the subarachnoid space at L2 level.

notochord can be observed only in the center of nucleus pulposus in adults. It is certain that chordomas arise from vertebral body, not intervertebral disc, which includes primitive notochord remnants. Ulich and Mirra demonstrated the remnants of notochord and hyaline cartilage in the vertebral body and advocated that chordomas could arise from these remnants ${ }^{14}$.

These tumors are slow growing and the symptoms are closely related with the localization of the tumor and to the tumor's compression to the root, spinal cord and paravertebral tissues. Compression of anterior column and root are predominant, parestesia and pain are most common complaints in patients with lumbar chordomas and usually takes so long time before any other neurological symptoms develop. Our patient was complaining only for low back pain for last one year. Nocturnal back pain, that not respon-

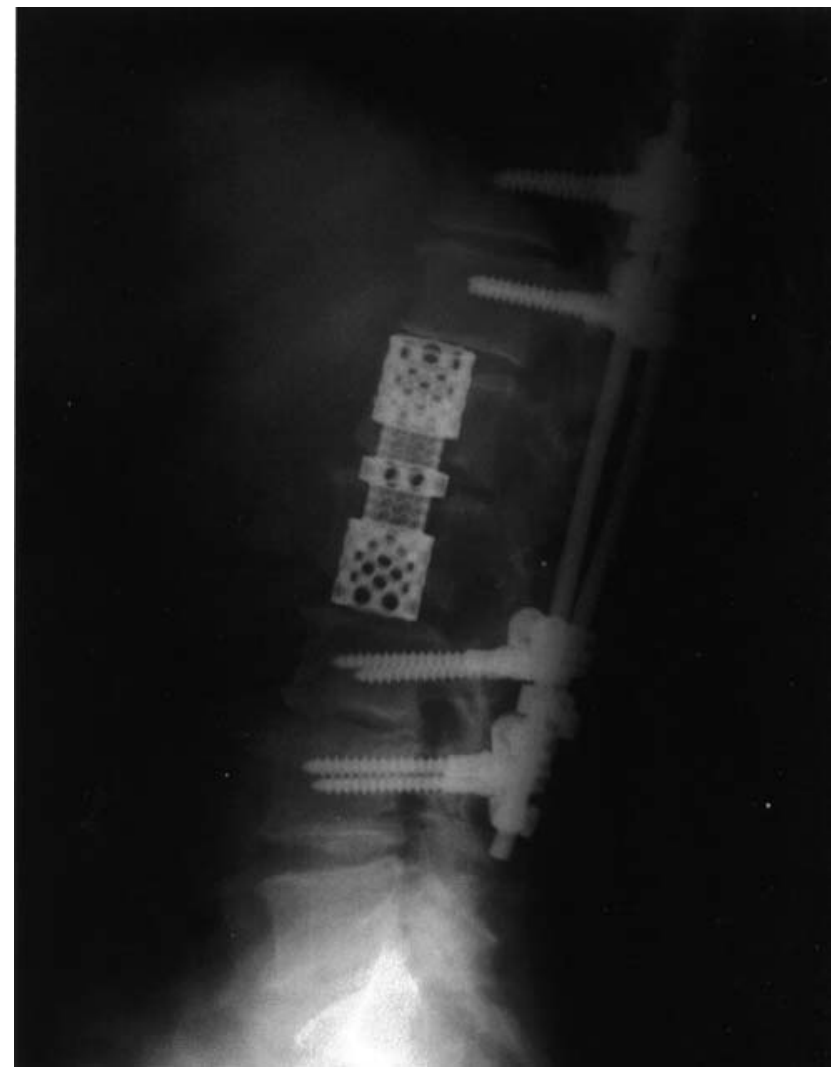

Figure 2. Postoperative X-ray showing Th 12 to L3 interbody fusion and stabilization after resection of tumor.

ding to the bed rest, is typical ${ }^{9}$. These lesions frequently cause motor changes due to spinal cord or root compression, but paraplegia rarely occurs as a long term complication ${ }^{11}$. $40 \%$ of patients have a trauma history with the same level of the tumor, while our patient denied such a history ${ }^{13}$.

Because of their location, lumbar chordomas may easily be confused with more common tumors in the lumbar spine such as aneurysmal bone cyst, giant cell tumor, hemangioma, myeloma and metastasis, and preoperative diagnosis can be difficult ${ }^{8}$.

The radiographic features of the chordomas do not depend on the anatomic location of the lesion. Vertebrae generally appears irregularly because of the destruction and expansive because of the slow growing of the lesion and remodeling and reactive bone formation ${ }^{11}$. Anterior soft tissue mass of the involved vertebrae is the most important radiological finding and shows that prevertebral involvement is greater than osseous involvement ${ }^{13}$. Myelographic investigations were performed before MRl to demonstrate the epidural involvement of the tumor ${ }^{13}$. CT is useful to identify the soft tissue tumor, to demonstrate the calcifications, osseous destruction and especially, if combined with intrathecal contrast material, epidural involvement. Chordomas show calcification with the ratio of $40-80 \%$. 
At present, MR imaging is definitely the method of choice for the diagnosis and preoperative assessment of spinal column chordomas. Spinal chordomas are most commonly iso- or hypointense on $\mathrm{T}_{1}$-weighted images, and moderately hyperintense on $\mathrm{T}_{2}$-weighted images ${ }^{4}$. The case presented here represents good example of the MR imaging findings of spinal chordomas. On $\mathrm{T}_{\mathrm{I}}$-weighted and $\mathrm{T}_{2}$-weighted $\mathrm{MR}$ images, a iso-intensity and high-intensity signal, respectively, were revealed.

Chordomas are semi-liquid, usually soft, green and transparent tumors. Although they are well demarcated from soft tissue, they can not be dissected from osseous tissue. They often contain focal calcifications, ossifications, hemorrhage, necrosis and cyst formations. The characteristic histopathological features of these tumors are vacuoled cells containing intracytoplasmatic mucus which is called physaliferous ${ }^{6}$. Differential diagnosis includes chondrosarcomas and adenocarcinomas ${ }^{11}$.

Surgical resection, radiation therapy, and chemotherapy are the current proposed therapy modalities for the spinal chordomas. The cure could be achieved by total removal and it must be the aim of therapy. Because of late appearance of clinical symptoms, when the tumor has been reached the extensive size, the surgical management of spinal chordomas becomes a challenge to surgeon. Surgical protocols for spinal chordomas are developed with the experience with spinal column tumors. Laminectomy was performed in the past years in the treatment of spinal chordomas and decreased paraplegia rates, pain and cord compression. The current surgical protocol for spinal chordomas consists of total removal of the lesion with a wide margin, spinal decompression, reconstruction and stabilization of the spine at the same time. Tumor spreading must be avoided during surgery. Recurrence will be definitely occurs if the removal is subtotal. In our patient, radical resection of the tumor resulted in clear improvement of symptoms. The actual malignant behavior of the chordomas is dependent on local aggressiveness, high recurrent rates and invasiveness to the important adjacent structures. Recurrent tumors can be treated with local excision, which can achieve good palliation, or with radiotherapy ${ }^{7,13}$.

Spinal chordomas usually spread by hematogeneously ${ }^{11}$. Because of survival largely depends on success or failure of complete excision of tumor, there is no direct relationship between the metastasis and survival ${ }^{13}$. The metastasis rates of chordomas differ from its location. While metastasis rate is $9 \%$ for intracranial chordomas, it is more common for sacrococcygeal and lumbar chordomas than intracranial tumors. Lumbar chordoma's metastasis rate is the highest ${ }^{10}$. The most common site of metastasis is lung and lymph nodes. Liver and bone metastasis are also common. Plain chest X-ray and bone scintigraphy must be assessed because of the possibility of metastasis to the lung for bone metastasis ${ }^{11}$. There were no sign of metastasis in his chest $\mathrm{X}$-ray and bone scintigraphy of our patient at his first evaluation and last follow-up.

Reports have failed to show any benefit from chemotherapy. Although chordomas are considered to be radioresistant, adjuvant radiotherapy has a place after subtotal resection and recommended as an adjuvant therapy ${ }^{1}$. As postoperative radiotherapy has a favourable influence on recurrence-free interval and survival, our patient received radiotherapy following surgery.

For spinal chordomas, the lower spinal level means the longer survival and the mortality rate of lumbar chordoma is better than that for thoracic or cervical lesions. The 5year survival rate for patients with vertebral chordomas is approximately $50 \%$, and with effective treatment the disease-free interval at 5 years is 30 to $50 \% 0^{3,12,13}$.

\section{Conclusion}

Lumbar spine is an uncommon localization for chordoma. With the aid of modern imaging technique, that make possible diagnosis of spinal chordoma preoperatively, modern surgical techniques, that permit complete removal of spinal chordomas, can significantly improve local tumor control, increase survival. We agree the other authors in emphasizing the importance of completely excising the spinal chordoma which is followed by postoperative radiotheraphy.

\section{References}

1. Amendola, B.E., Amendola, M.A., Oliver, E., et al.: Chordoma: role of radiation therapy. Radiology. 1986; 158: 839-43.

2. Baratti, D., Gronchi, A., Pennaechioli, E., et al.: Chordoma: natural history and results in 28 patients treated at a single institution. Ann Surg Oncol. 2003; 10: 291-296.

3. Bjornsson, J., Wold, L.E., Ebersold, M.J., et al.: Chordoma of the mobile spine. A clinicopathologic analysis of 40 patients. Cancer. 1993; 71: 735-740.

4. Bosma, J.J., Pigott, T.J., Pennie, B.H., et al.: En bloc removal of the lower lumbar vertebral body for chordoma. Report of two cases. J Neurosurg. 2001; 94 (2 Suppl): 284-291.

5. Chambers, P.W., Schwinn, C.P.: Chordoma. A clinicopathologic study of metastasis. Am J Clin Pathol. 1979; 72: 765-776.

6. Dahlin, D.C., MacCarty, C.S.: Chordoma. Cancer. 1952; 5:1170-1178.

7. Dahlin, D.C.: Bone Tumors. General Aspects and Data on 8,542 Cases. Springfield, I11: Charles C. Thomas, 1986. pp 379-393.

8. Hartofilakidis-Garofalidi, Papathanassiou, B.T., Kambouroglou, G.: Chordoma of the lumbar spine. Int Surg. 1968; 
50: 566-570.

9. Healey, J.H., Lane, J.M.: Chordoma: a critical review of diagnosis and treatment. Orthop Clin North Am. 1989; 20: 417-426.

10. Higinbotham, N.L., Phillips, R.F., Farr, H.W., et al.: Chordoma. Thirty-five-year study at Memorial Hospital. Cancer. 1967; 20: 1841-1850.

11. Mindell, E.R.: Chordoma. J Bone Joint Surg Am. 1981; 63: 501-505.

12. Sundaresan, N., Galicich, J.H., Chu, F.C.: Spinal chordomas. J Neurosurg. 1979; 50: 312-319.

\section{Comentario al Trabajo: Chordoma of the lumbar spine: a case report de Tuna y cols.}

I agree with the autors that chordoma is a malignant bone tumor that grows slowly, often recurs locally, and metastasizes later. Although different treatment approaches exist, including radiation and surgery, the most effective treatment is early and complete surgical tumor excision. Immediate spinal stability must be achieved with rigid fixation and bone graft.

Authors used a corpectomy and tumorectomy with Thl2L2 interbody fusion and stabilization procedure. Combined (two-stage) anterior-posterior approach including an anterior retroperitoneal approach allows en-bloc resection of lumbar vertebral bodies, that it could avoid recurrences. En-bloc resection is technically demanding, but potentially curative.

Surgery combined with radiation-therapy may cause tumor regression that lasts for many years. Proton beam radiation therapy may be especially effective in controlling local disease in many cases of incomplete surgical resection.

\section{S. Martín-Ferrer}

Girona
13. Sundaresan, N.: Chordomas. Clin Orthop. 1986; 204: 135-142.

14. Ulich, T.R., Mirra, J.M.: Ecchordosis physaliphora vertebralis. Clin Orthop. 1982; 163: 282-289.

Tuna, H.; Aydin, V.; Bozkurt, M.; Attar, A.: Chordoma of the lumbar spine: a case report. Neurocirugía 2005; 16: 169-172.

Corresponding Author: Hakan Tuna MD 3624 Beckham Dr, Shreveport, LA 71104, USA. 